\title{
Gold nanoparticle: synthesis and characterization
}

\author{
Harihar Nath Verma ${ }^{1}$, Praveen Singh ${ }^{2}$ and R. M. Chavan ${ }^{1}$
}

1. Animal Biochemistry Section, Indian Veterinary Research Institute, Izatnagar - 243122, Bareilly, Uttar Pradesh, India; 2. Incharge-Biophysics and Electron Microscopy Section

Indian Veterinary Research Institute, Izatnagar - 243122, Bareilly, Uttar Pradesh, India

Corresponding author: Harihar Nath Verma, Mob: +919458753028, email:drhnverma@gmail.com

Received: 27-11-2013, Revised: 03-01-2014, Accepted: 06-01-2014, Published online: 15-02-2014

doi: $10.14202 /$ vetworld.2014.72-77

How to cite this article: Verma HN, Singh P and Chavan RM (2014) Gold nanoparticle: synthesis and characterization, Veterinary World 7(2): 72-77.

\begin{abstract}
Aim: The aim of present study was to synthesize and characterize gold nanoparticles applicable in therapeutics and diagnostics tool for different diseases in particular for peste des petits ruminants (PPR).

Materials and Methods: Gold nanoparticles were synthesized by the reduction of $\mathrm{Au}^{+3}\left(\mathrm{HAuCl}_{4}\right)$ to $\mathrm{Au}^{0}$. For this purpose, the reduction reaction between tetrachloroauric acid $\left(\mathrm{HAuCl}_{4}\right)$ and trisodium citrate $\left(\mathrm{Na}_{3} \mathrm{C}_{6} \mathrm{H}_{5} \mathrm{O}_{7} .2 \mathrm{H}_{2} \mathrm{O}\right)$ was used in an aqueous solution. The initial gold concentration, trisodium citrate concentration and temperature were changed and their effects on particle size and size distribution were observed. At the end of the reduction reaction, gold nanoparticles with narrow size distribution were obtained. Zeta sizer and transmission electron microscopy (TEM) were used to characterize and determine the size and size distribution of the synthesized gold nanoparticles.
\end{abstract}

Results: In the present work optimal gold salt concentration, trisodium citrate concentration and temperature were found to be $20 \mathrm{mM}, 1.5 \%$ and $97^{\circ} \mathrm{C}$ respectively. The morphology, size, and shape of synthesized gold nanoparticles were characterized by using spectrophotometry, transmission electron microscopy (TEM) and zeta sizer. In our study colloidal gold nanoparticle of $15-20 \mathrm{~nm}$ size were obtained.

Conclusions: The uniform gold nanoparticles were synthesized by the citrate reduction method. The effects of various experimental parameters (Gold salt concentration, trisodium citrate concentration and mixing rate) on its size and size distribution at optimum $\mathrm{pH}$ and temperature were investigated. The gold nanoparticle synthesized by this method has been used for the preparation of gold-labeled lateral flow strip for peste des petits ruminants (PPR) diagnosis.

Key words: chemical reduction, gold nanoparticles, sodium citrate, tetrachloroauric acid

\section{Introduction}

Nanotechnology is an anticipated manufacturing technology that allows the long-established trend toward smaller, faster, cheaper materials and devices. Gold nanoparticles (GNPs) are the most compatible nanomaterial for preparation of engineered nanoplatforms in smart sensing devices. Surface Plasmon resonance property of GNP makes them most suitable engineered nanomaterial for bioimaging, biomedical therapeutics and biodiagnostic tools [1]. GNPs, also named as gold colloids, have attracted increasing attention due to their unique properties in multi disciplinary research fields [2,3]. Although GNPs are defined by tiny size, significant quantities of GNPs are likely required in many commercial and industrial applications. Remarkably, novel emerging applications bring a huge growth of the global demand of GNPs. For instance, (a) biomolecule- and/or biopolymer-conjugated GNPs are largely used as biomarkers and biodelivery vehicles in the medicine/ pharmacy and in cosmetic products. GNPs are employed as anti-aging components for skin protection [4]. (b)

Copyright: The authors. This article is an open access article licensed under the terms of the Creative Commons Attribution License (http://creativecommons.org/licenses/by/2.0) which permits unrestricted use, distribution and reproduction in any medium, provided the work is properly cited.
GNPs are used to treat wool or cotton fibres for a permanent coloration of value textiles. (c) Various polymer/gold nanocomposites display a high potential for novel coatings and paintings [5,6]. (d) GNPs are used to enhance the performance of non-volatile memory devices [7] and low temperature printing metal inks in electronics [8].(e) GNPs as catalysts are developed in novel usages $[9,10]$. Gold nanoparticles with diameter from 15-20 nm can be generated by reduction of auric-chloride with trisodium citrate [11]. The gold nanoparticles in $15-20 \mathrm{~nm}$ size range have attracted attention for fabrication of smart sensing devices in biomedical sciences as diagnostic tools. Citrate capped GNPs are negatively charged, which can be exploited for electrostatic interactions with some positively charged biomolecules like antibody. The compatibility of GNPs is excellent with antibody or antigen and other biomolecules; moreover, GNPs do not affect the functional activity even after immobilization. This in turn can be used for the detection of target analyte specifically. Therefore, surface fictionalization of gold nanoparticles could accelerate antibody-antigen reaction, which further amplify the signal in immunoassay [12]. Due to all above advantages, GNPs were used in development of lateral flow assay, which is one-step on-site screening test for analyte. Therefore, 


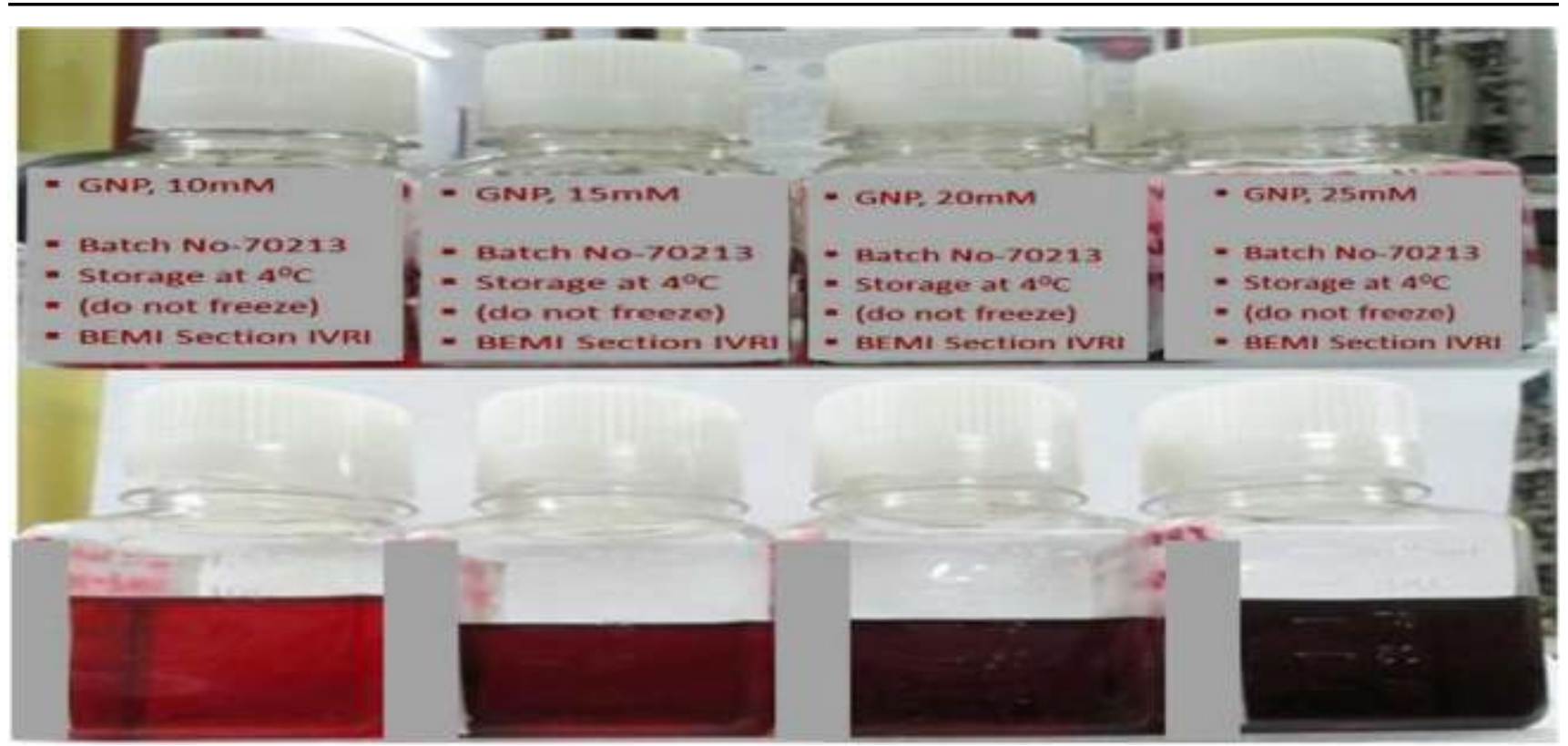

Figure-1. Color variation of gold nanoparticle solutions of 10, 15, 20, 25 mM concentration

more attention should be paid on effective synthesis methods to match the enlarging demand of GNPs. In the past decades, though many synthetic strategies have been developed to prepare GNPs in organic or aqueous solvents $[13,14]$, the citrate reduction method has remained the best option to fit the enlarging demand of GNPs due to advantages such as inexpensive reductant, non-toxic water solvent, and low pollution in the reaction [15]. The simple operation of pouring rapidly a certain amount of trisodium citrate solution into a boiling solution of 10-25 mM chloroauric acid produces narrowly distributed GNPs, which are biocompatible and easily handled in applications $[16,17]$. So, this method is extensively used in GNP-based bioassays and biomedicine systems [18] and even in structured/ assembled nanomaterials $[19,20]$. In the pioneering work on the citrate reduction method, Turkevich in 1951 reported the basic experimental approach and the effect of temperature and reagent concentration upon the nanoparticle size and size distribution and in 1973; Frens [21] published the control of size variation of GNPs by changing the concentration of sodium citrate. Recently, the decisive role of trisodium citrate on the $\mathrm{pH}$ value of the reaction mixture and the nanoparticle size was demonstrated based on experimental and theoretical modelling results [22]. On the other hand, in the majority of the published citrate reduction works, GNPs were synthesized from a dilute solution of $0.25 \mathrm{mM}$ tetrachloroauric acid $\left(\mathrm{HAuCl}_{4}\right)$; such a concentration yields aqueous GNPs with low weight content $(0.005 \%)$ as a disadvantage. Herein, to meet the need of high concentrations, we synthesized uniform GNPs from 10, 15, $20 \mathrm{mM} \mathrm{HAuCl}$ solutions. More excellent featured structures can be synthesized. Arising of some disciplinary like nanoengineering [2], nanoelectronic [3], and nanobioelectronic [4] forced the synthesis of suitable sized and functional block structures.

\section{Materials and Methods}

Materials: The chemicals used in this study were mostly dried and were prepared according to requirements. The auric-chloride ( $\mathrm{HAuCl} 4)$ was procured from $\mathrm{CDH}$ (India), trisodium citrate from Sigma (USA), bovine serum albumin (BSA) from Amresco (USA), polyethylene glycol (PEG) from Merck (India), sodium azide $\left(\mathrm{NaN}_{3}\right)$ from Spectrochem (India), sodium chloride $(\mathrm{NaCl})$ and tris hydrochloride from SD fine chemicals (India), potassium dihydrogen phosphate $\left(\mathrm{KH}_{2} \mathrm{PO}_{4}\right)$ from Loba chemie (India).

Gold nanoparticles synthesis: Synthesis of gold nanoparticles was done with citrate reduction method $[11,21]$. A series of nanoparticles were synthesized using different dilutions. The quantity of $200 \mu \mathrm{l}, 300$ $\mu 1,400 \mu \mathrm{l}$ and $500 \mu \mathrm{l}$ was taken from $1 \%$ tetrachloroauric acid (with $49 \% \mathrm{Au}$ ) solution and diluted to $50 \mathrm{ml}$ to make $10 \mathrm{mM}, 15 \mathrm{mM}, 20 \mathrm{mM}$ and $25 \mathrm{mM}$, aqueous solutions of tetrachloroauric acid respectively. This solution in beaker was boiled until the temperature reach $97^{\circ} \mathrm{C}$ on a hot plate with magnetic stirrer. To this boiling solution, $1.5 \%$ trisodium citrate was added in continuous mode quickly with simultaneous stirring. After addition of sodium citrate solution, stirring continued until solution turns brilliant red colored as shown in Figure-1. The synthesis reaction summarised as given below.

$2 \mathrm{HAuCl}_{4}+3 \mathrm{C}_{6} \mathrm{H}_{8} \mathrm{O}_{7}$ (citric acid) $\rightarrow 2 \mathrm{Au}+3 \mathrm{C}_{5} \mathrm{H}_{6} \mathrm{O}_{5}(3-$ ketoglutaric acid $)+8 \mathrm{HCl}+3 \mathrm{CO}_{2}$

This solution was stored at $4^{\circ} \mathrm{C}$ for further use.

The submitted work investigates gold nanoparticles synthesis by reduction reaction between tetrachloroauric acid and trisodium citrate. The dependence of nanoparticle size and size distribution were tested by changing the concentrations of gold salt, trisodium citrate, temperature and mixing rate. The nanoparticles were characterized by zeta sizer to 


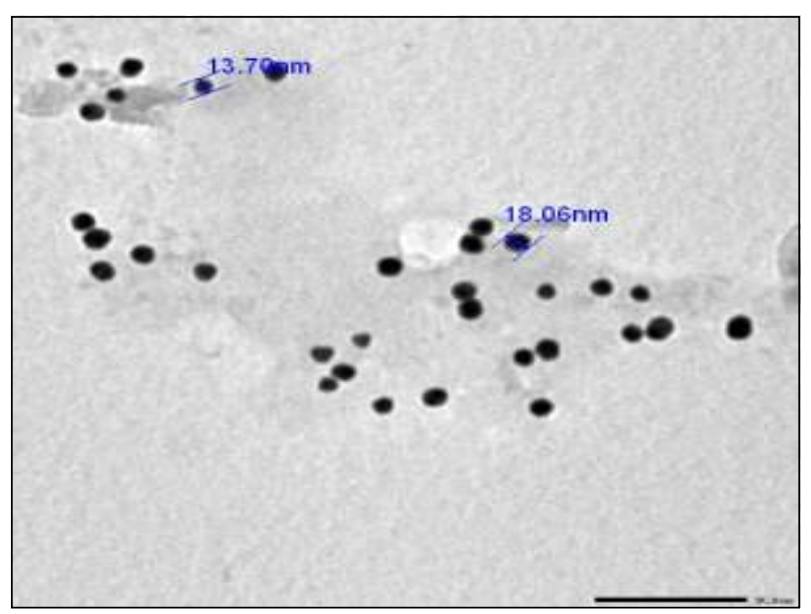

Figure-2. TEM I mages of colloidal gold solution

determine electrical charge, size and size distributions.

Gold salt concentration: In this part of the study, the gold salt initial concentration effect was investigated. Gold salt solution was mixed with deionised water $(\mathrm{pH}$ 7.2) at different ratios. Therefore, gold salt concentrations were changed from $10 \mathrm{mM}$ to $25 \mathrm{mM}$. The reducing agent concentration trisodium citrate, $1.5 \%$ ) and mixing rate were kept constant during synthesis experiments.

Trisodium citrate concentration: The effect of trisodium citrate, which was used as reducing agent to the nanoparticle size, was investigated in this part of the study. Triodium citrate concentration was changed from $0.5 \%$ to $2 \%$. Gold salt concentration $(20 \mathrm{mM})$, temperature, and mixing rate were kept constant during experiments.

The mixing rate and nanoparticle size: In this part of the study, the mixing rate effect to the nanoparticle size and size distribution was investigated. For this purpose, the mixing rate was changed and optimised. Other parameters, such as gold salt concentration temperature, and trisodium citrate concentration, were kept constant as $20 \mathrm{mM}$ and $1.5 \%$, respectively.

Gold nanoparticle characterization: The morphology, size, and shape of synthesized gold nanoparticles were characterized using transmission electron microscopy, zeta sizer and spectrophotometer. The transmission electron microscopic measurements were performed on JEOL, JEM-1400, HSADL at Bhopal at an acceleration voltage of $120 \mathrm{KV}$. A drop of colloidal gold solutions from $10 \mathrm{mM}, 15 \mathrm{mM}$, and $20 \mathrm{mM}$ were placed on carbon coated TEM copper grid. The film was allowed to dry for 5 minutes and the excess solution was removed using a blotting paper. The size distribution of particles was measured from enlarged images of transmission electron microscopy (TEM) by taking different counts for each sample.

The hydrodynamic diameter of nanoparticles was measured in triplicates using zeta sizer nano-ZS (Malvern instrument, UK). The data analysis was performed in automatic mode and measured sizes were presented as average value of 30 runs. The zeta potential measurements of nanoparticles were measured on a zeta sizer nano-ZS (Malvern instruments, UK). Thirty runs were carried out in triplicates and the average values were estimated by smoluchowski approximation from the electrophoretic mobility. This study was conducted for both bare gold nanoparticles and also for PPR antibody conjugated gold nanoparticles. The UVVisible spectroscopic measurement was monitored using single beam spectrophotometer (Systronics 169) at different wavelength $(200-700 \mathrm{~nm})$.

\section{Results and Discussion}

Synthesis and characterization: The colloidal gold solution with size range of 5 to $60 \mathrm{~nm}$ particle size are stable for long duration in absence of any special stabilizing agent [23]. The chloroauric acid $(20 \mathrm{mM})$ was reduced to gold atoms using sodium citrate $(1.5 \%)$ and many colloidal gold particles $(18 \mathrm{~nm})$ were accumulated in solution [11,21]. The temperature $\left(97^{\circ} \mathrm{C}\right)$, ratio of gold to citrate, and the order of addition of reagents control the size distribution of gold nanospheres generated by this method [24]. These particles were characterized for size and potential for their optimum properties.

Transmission electron microscopy: Fig. 2 shows the transmission electron microscopy (TEM) image of gold nanoparticles with different concentration and size distribution of well-dispersed suspensions. The size of gold nanoparticles has been determined by measuring the diameter of whole particles on TEM images. The average diameter of colloidal gold was in the range of $18 \mathrm{~nm}$ with very few particles of higher and lower size distribution. The TEM image show that the gold colloid is in monodispersional state, this is because of negatively charged layer of citrate ions, which repel from each other. This monodispersity accounts for the probe preparation and generation of color signal in chromatographic strip assay. Moreover, the TEM images show that most of the gold nanospheres are round or spherical in shape.

UV-VIS spectrophotometry: Spectrophotometry is 


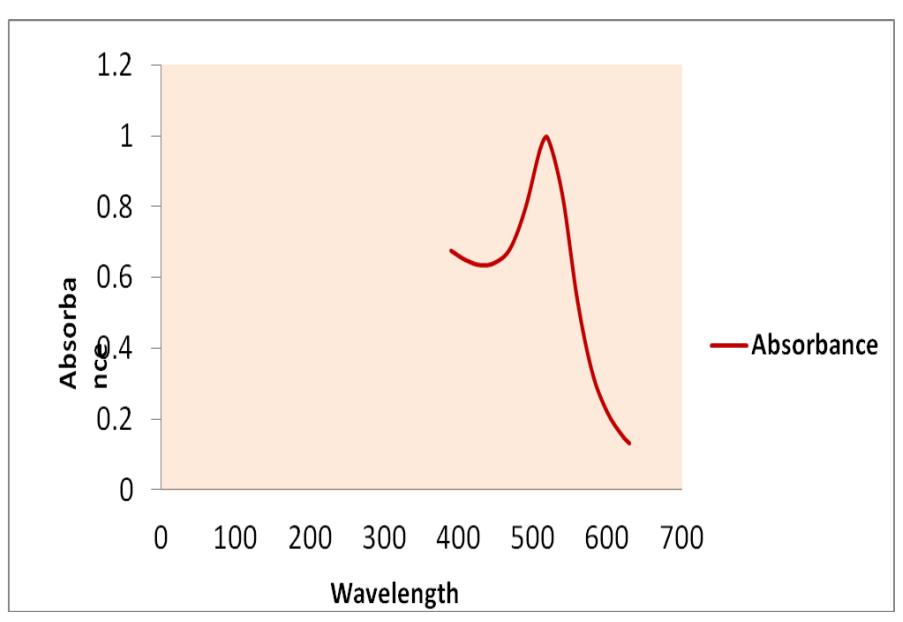

Figure-3. Absorbance of gold nanoparticles at $520 \mathrm{~nm}$

Size Distribution by Intensity

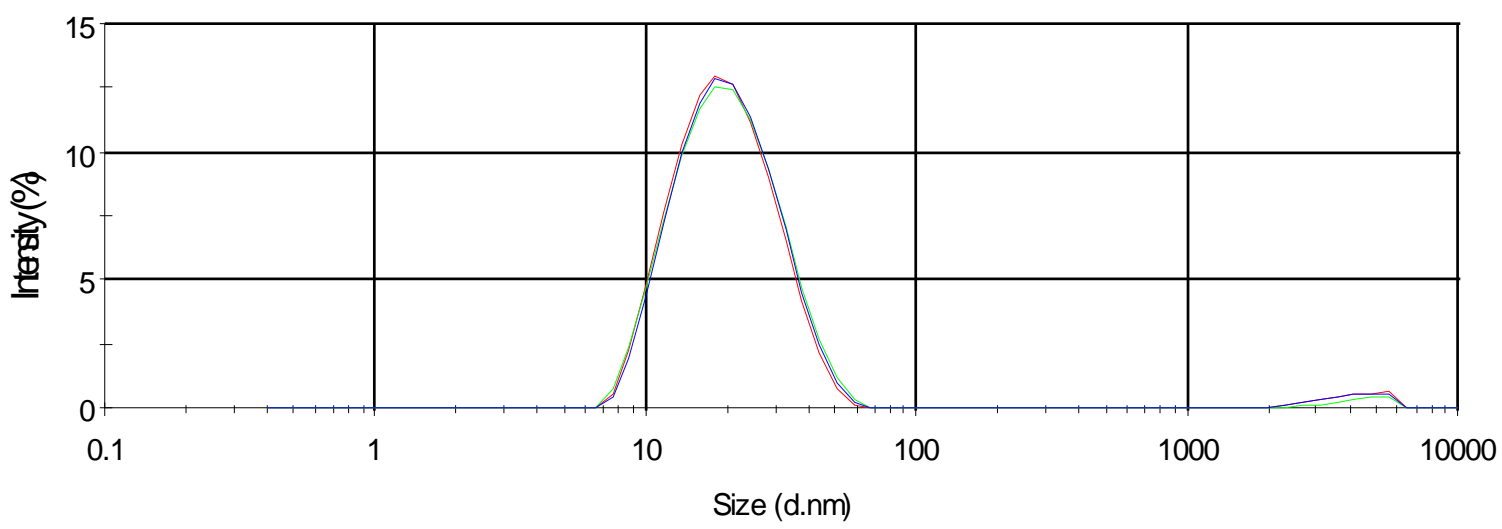

Figure-4. Zeta Size distribution of gold nanoparticles

another important aspect for characterization of gold nanoparticles. With increase in particle size, the absorption peak shifts to longer wavelength and the width of absorption spectra is related to the size distribution range. Generally, gold nanospheres display a single absorption peak in the visible range between 510-550 nm, because of surface Plasmon resonance and show heavy absorption of visible light at $520 \mathrm{~nm}$. This gives brilliant red color to Gold Nanoparticle (GNP), which varies according to their size. In present study the absorption of gold nanoparticle was measured in single beam spectrophotometer and absorption maxima was noted at different wavelength (390-630 $\mathrm{nm})$. The colloidal gold synthesized in experiment shown heavy absorption at $520 \mathrm{~nm}$ as in Fig. 3. The absorbance at $520 \mathrm{~nm}$ was 1.368 .

Zeta size and zeta potential distribution: The zeta study was conducted for particle size, size distribution as well as for zeta potential measurement of both bare and antibody conjugated gold nanoparticles. In transmission electron microscopy (TEM) study, images show particles with lower and higher size range. To obtain uniformity of size, colloidal gold solution was centrifuged (at $8000 \mathrm{rpm}$ ) for 10 minutes. The supernatant was collected and characterized for further use. Table-1 shows the size distribution of colloidal gold particles.

For zeta potential distribution study, peak number and peak area gives important explanation. Three cycles of different counts were run and average of the counts was taken. The peak mean gives the mean diameter of particle and peak area gives the percentage of mean diameter according to intensity. The graphs were plotted using the means of all peaks mean diameter and the intensity of peak area as shown in the Fig. 4.

In present study, it has found that $20 \mathrm{mM}$ centrifuged sample have average zeta of $18 \mathrm{~nm}$ with peak 1 mean of $21 \mathrm{~nm}$ of $97 \%$ intensity. Whereas, the peak 2 area show only 2-3\% of particles. This indicates that, most of the particles have average diameter of 18 $\mathrm{nm}$ and are excellent for development of the probe. Beside size distribution, the zeta potential measurement is also important for characterization. The negative charge on nanoparticles due to citrate ions is another important indicator for particle size [25]. The negative charge indicates that the particle size is 
Table-1. Zeta Size distribution of gold nanoparticles

\begin{tabular}{lccccccccc}
\hline Record Sample name & z-ave d.nm & Pdi & Peak1 mean & Peak2 mean & Peak3 mean & Peak1 area\% & Peak2 area\% & Peak3 area\% \\
\hline 79 & $20 \mathrm{mM}$ & 18.02 & 0.217 & 20.87 & 4181 & 0 & 97.2 & 2.8 & 0 \\
80 & $20 \mathrm{mM}$ & 18.08 & 0.212 & 21.46 & 4464 & 0 & 98.3 & 1.7 & 0 \\
81 & $20 \mathrm{mM}$ & 18.27 & 0.223 & 21.37 & 4169 & 0 & 98.3 & 2.7 & 0 \\
\hline
\end{tabular}

Zeta Potential Distribution

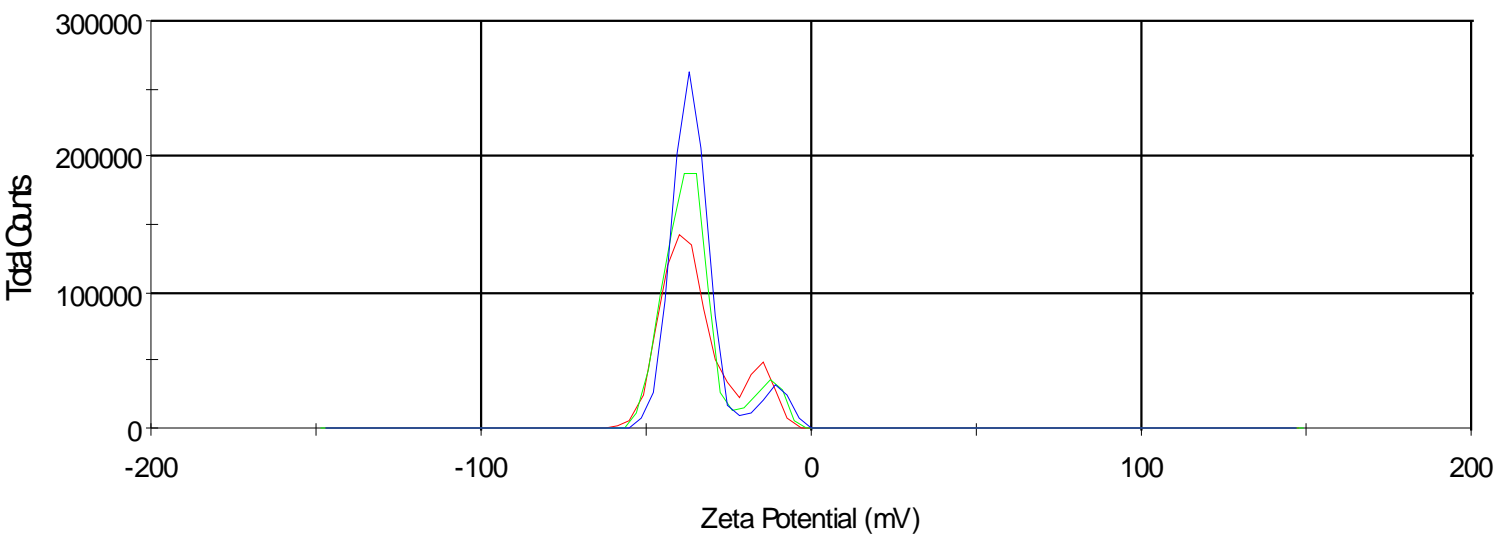

Record 91: $20 \mathrm{mM} 1.5 \% 1$

Record 92: $20 \mathrm{mM} 1.5 \% 2$

Figure-5. Zeta Potential of Bare Gold Nanoparticles (20 mM)

smaller than $100 \mathrm{~nm}$. In present study, zeta potential of the synthesized nanoparticles was highly negative Fig. 5 .

Conjugation of PPR antibody with colloidal gold nanoparticles synthesized by this method was very good for preparation of PPR diagnostic strips. Gold nanoparticle labelled immuno-chromatographic strip test is a promising candidate for diagnostic application at field condition even for non-expert users. At present time many department of IVRI and other research institute use gold particles as labelling material in therapeutics and diagnostics tool for different diseases.

\section{Conclusion}

The present work was carried out to investigate the synthesis and characterization of gold nanoparticles and the effect of various experimental parameters (temperature, gold salt concentration trisodium citrate concentrations, mixing rate and $\mathrm{pH}$ ) on its size and size distribution. The uniform gold nanoparticles with small size can be synthesized from the tetrachloroauric acid precursor at high concentration by the citrate reduction method. The optimal gold salt concentration, trisodium citrate concentration, $\mathrm{pH}$ and temperature were found to be $20 \mathrm{mM}$, $1.5 \%, 7.2$ and $97^{\circ} \mathrm{C}$ respectively. The morphology, size, and shape of synthesized gold nanoparticles were characterized using transmission electron microscopy, zeta sizer and spectrophotometer. In the study colloidal gold nanoparticle of $15-20 \mathrm{~nm}(18 \mathrm{~nm})$ size were obtained. Narrow size distribution and small monosize gold nanoparticles also offer advantages for selfassembled monolayer formation and enhanced surface area. Such environmental-friendly synthesis method for gold nanoparticles may have a great potential in large-scale manufacturing to match the increasing commercial and industrial demands.

\section{Authors' contributions}

HNV and PS designed the work plan. HNV did all the laboratory work. RMC helped in photography and characterisation of gold nanoparticle. PS and HNV drafted the manuscript and revised it. All authors read and approved the final manuscript.

\section{Acknowledgements}

The authors gratefully acknowledge the financial support provided by Indian Council of Agricultural Research, New Delhi, India and The Director of Indian Veterinary Research Institute, Izatnagar, India.

\section{Competing interests}

The authors declare that they have no competing interests.

\section{References}

1. Jain, P.K., Lee, K.S., El-Sayed, I.H. and EL-Sayed, M.A. (2006) Calculated absorption and scattering properties of gold nanoparticles of different size, shape, and composition: application in biological imaging and biomedicine. J. Phys. Chem. B., 110: 7238-7248.

2. Daniel, M.C. and Astruc, D. (2004) Gold nanoparticles: Assembly, supramolecular chemistry, quantum-size-related properties and applications toward biology, catalysis, and nanotechnology. Chem. Rev., 104: 293-346.

3. Sardar, R., Funston, A.M., Mulvaney, P. and Murray, R.W. (2009) Gold Nanoparticles: Past, Present, and Future. Langmuir., 25: 13840-13851.

4. Boisselier, E. and Astruc, D. (2009) Gold nanoparticles in nanomedicine: preparations, imaging, diagnostics, therapies and toxicity. Chem. Soc. Rev., 38: 1759-1782. 
5. Freudenberger, R., Zielonka, A., Funk, M., Servin, P., Haag, R., Valkova, T. and Landau, U. (2010) Recent developments in the preparation of nano-gold composite coatings. Gold Bull., 43: 169.

6. Jans, H., Jans, K., Lagae, L., Borghs, G., Maes, G. and Huo, Q. (2010) Poly (acrylic acid)-stabilized colloidal gold nanoparticles: synthesis and properties. Nanotechnol., 21: 455702 .

7. Li, D.X., Li, C.F., Wang, A.H., He, Q. and Li, J.B. (2010) Hierarchical gold/copolymer nano-structures as hydrophobic nanotanks for drug encapsulation. J. Mater. Chem., 20: 7782.

8. Bishop, P.T., Ashfield, L.J., Berzins, A., Boardman, A., Buche, V., Cookson, J., Gordon, R.J., Salcianu, C. and Sutton, P.A. (2010) Printed gold for electronic applications. Gold Bull., 43: 181.

9. Wallace, W.T. and Whetten, R.L. (2002) Coadsorption of CO and $\mathrm{O}_{2}$ on selected gold clusters: Evidence for efficient roomtemperature $\mathrm{CO}_{2}$ generation. J.Am. Chem. Soc., 124: 7499.

10. Zhou, X.C., Xu, W.L., Liu, G.K., Panda, D. and Chen, P. (2010) Size-Dependent Catalytic Activity and Dynamics of Gold Nanoparticles at the Single-Molecule Level. J. Am. Chem. Soc., 132: 138.

11. Turkevich, J., Stevenson, P.C. and Hillier, J. (1951) A study of the nucleation and growth processes in the synthesis of colloidal gold. Discuss Faraday Soc., 11:55-75.

12. Richars, G.D. (1996) Preparation of colloidal gold particles of various sizes using sodium borohydride and sodium cyanoborohydride. Anal. Biochem., 23: 168-170.

13. Martin, M.N., Basham, J.I., Chando, P. and Eah, S. K. (2010) Charged Gold Nanoparticles in Non-Polar Solvents: 10-min Synthesis and 2D Self-Assembly. Langmuir., 26: 7410.

14. Volkert, A.A., Subramaniam, V. and Haes, A.J. (2011) Implications of citrate concentration during the seeded growth synthesis of gold nanoparticles. Chem. Commun (Camb), 47: 478-480.

15. Ji. X.H., Song, X.N., Li, J., Bai, Y.B., Yang, W.S. and Peng, X.G. (2007) Size control of gold nanocrystals in citrate reduction: The third role of citrate. J. Am. Chem. Soc., 129: 13939-13948.

16. Nguyen, D.T., Kim, D.J., So, M.G. and Kim, K.S. (2010) Experimental measurements of gold nanoparticle nucleation and growth by citrate reduction of $\mathrm{HAuCl}_{4}$. Adv. Powder Technol., 21: 111-118.

17. Uppal, M.A., Kafizas, A., Ewing, M.B. and Parkin, I.P. (2010) The effect of initiation method on the size, monodispersity and shape of gold nanoparticles formed by the Turkevich method. New J. Chem., 34:2906.

18. Kim, Y.P., Oh, Y.H. and Kim, H.S. (2008) Protein kinase assay on peptide-conjugated gold nano particles. Biosens. Bioelectron., 23:980-986.

19. Li, D.X., He, Q., Yang, Y., Möhwald, H. and Li, J.B. (2008) Two-stage $\mathrm{pH}$ response of poly (4 vinyl pyridine) grafted gold nanoparticles. Macromolecules., 41: 7254-7256.

20. Xia, H. and Wang, D. (2008) Fabrication of Macroscopic Freestanding Films of Metallic Nano-particle Monolayers by Interfacial Self-Assembly. Adv. Mater., 20: 4253-4256.

21. Frens, G. (1973) Controlled nucleation for the regulation of the particle size in monodisperse gold suspensions. Nat. Phys. Sci., 241:20-22.

22. Yang, S.C., Wang, Y.P., Wang, Q.F., Zhang, R.L. and Ding, B.J. (2007) UV irradiation induced formation of $\mathrm{Au}$ nanoparticles at room temperature: The case of $\mathrm{pH}$ values. Colloid Surf. A-Physicochem. Eng .Asp., 301: 174.

23. Safenkova, I.V., Zherdev, A.V., and Dzantiev, B.B. (2010) Correlation between the composition of multivalent antibody conjugates with colloidal gold nanoparticles and their affinity. J. Immunol. Methods., 357: 17-25.

24. Eustis, S. and El-Sayed, M.A. (2006) Why gold nanoparticles are more precious than pretty gold: Noble metal surface plasmon resonance and its enhancement of the radiative and nonradiative properties of nanocrystals of different shapes. Chem. Soc. Rev., 35(3): 209-217.

25. Amir, T., Fatma, A. and Hakan, A. (2009) Gold Nanoparticle Synthesis and Characterization. Hacettepe J. Bol. \& Chem. 37(3): 217-226.

$* * * * * * * *$ 